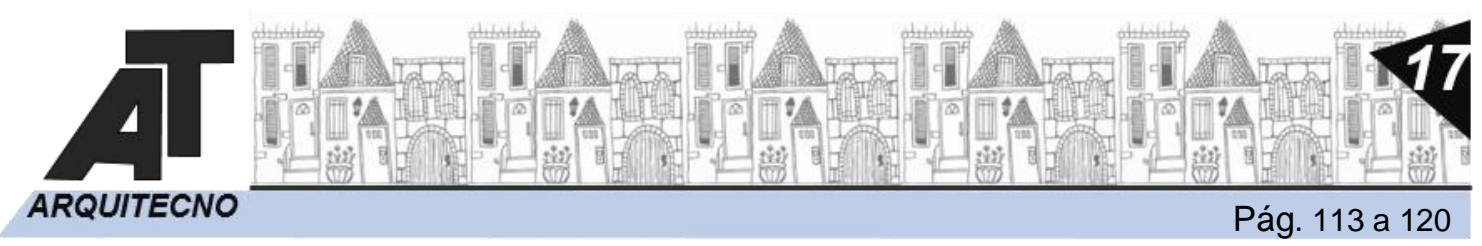

\title{
La extensión en Pandemia: la experiencia del Corralón Solidario
}

\section{Transfer in Pandemic: the Corralón Solidario experience}

Gustavo Cremaschi, María Elisa Cremaschi, María Eugenia Luna, Nelly Lombardi

Laboratorio de Tecnología y Gestión Habitacional, Facultad de Arquitectura y Urbanismo, Universidad de La Plata, La Plata, Argentina. corralonsolidario@fau.unlp.edu.ar

\section{RESUMEN}

En el marco de este artículo queremos compartir la experiencia realizada del Proyecto de Extensión "Corralón Solidario" de la Facultad de Arquitectura y Urbanismo de la Universidad Nacional de La Plata en el contexto pandémico provocado por el COVID 19.

El proyecto tiene como objetivo concientizar a la comunidad en general sobre los beneficios de recuperar residuos de construcción y demolición (RCD). Asimismo, busca contribuir al mejoramiento del hábitat a partir de optimizar recursos materiales y humanos en entornos solidarios por medio de la reutilización de RCD.

Frente a la situación de pandemia, surgió la necesidad de adecuar dicho proyecto a una nueva modalidad virtual, para ello diseñaron espacios sincrónicos y asincrónicos para capacitar virtualmente y reflexionar acerca de beneficios de recuperar estos materiales residuales de obras, siendo también un entorno propicio para detectar necesidades en el ámbito de los participantes y dar respuestas a las problemáticas emergentes.

\section{ABSTRACT}

In this article we want to share the experience of the "Corralón Solidario" Transfer Program of the Architecture and Urbanism School of the National University of La Plata in the COVID 19 pandemic context.

The program aims to raise awareness in the general community about the benefits of recovering Construction and Demolition Waste (C\&DW). Also, it seeks to contribute to the improvement of the habitat by optimizing material and human resources in supportive environments through the reuse of C\&DW.

In the pandemic situation, we had the need to adapt the program to a new virtual mode, for this were designed synchronous and asynchronous spaces to virtually train and consider the benefits of recovering these residual materials from works, being also a propitious environment to detect participants needs and provide answers to emerging problems.

PALABRAS CLAVES: RCD- CAPACITACIÓN VIRTUAL - UNIVERSIDAD - COVID19

KEY WORDS: C\&D - VIRTUAL TRAINING - UNIVERSITY - COVID19

FECHA DE RECEPCIÓN: 15/4/2021 | FECHA DE ACEPTACIÓN: 13/5/2021

DOI: http://dx.doi.org/10.30972/arq.0174994 


\section{INTRODUCCIÓN}

El "Corralón Solidario" de la Facultad de Arquitectura y Urbanismo de la Universidad Nacional de La Plata (FAU UNLP), nace en el año 2015 como un proyecto destinado a dar respuesta a una problemática crucial en la actividad de la construcción como es el destino de los residuos de obras de construcción y demolición $(\mathrm{RCD})$ a través de la recuperación y reutilización de materiales residuales, rezagos de obras o en desuso para destinarlos tanto a actividades de extensión universitaria como a proyectos. Frente a la situación de emergencia sanitaria que impone la pandemia provocada por el Coronavirus SARS-CoV-2 (COVID 19), se propone adecuar el abordaje del proyecto y repensar, hoy más que nunca, la relación con el medio ambiente y su demostrada incidencia con la salud de la sociedad.

La génesis propia del proyecto fomenta vinculaciones transversales con iniciativas que aborden la temática del hábitat facilitando así procesos de mejoramiento del mismo. En este sentido, el proyecto permite la retroalimentación permanente de la docencia, la investigación y la extensión a través de la construcción conjunta de talleres de capacitación y concientización para los vecinos/as de los Centros Comunitarios de Extensión Universitaria de la UNLP (CCEU) y la comunidad en general. Los CCEU son espacios construidos colectivamente entre la Universidad y la comunidad para abordar de manera conjunta los problemas de cada territorio.

De esta manera, se propone crear espacios donde se pueda capacitar virtualmente y reflexionar acerca de la eficiente reutilización y reciclado de materiales y su relación con la huella ecológica, proporcionando información y conocimientos importantes para detectar y diagnosticar las patologías más comunes en la construcción, siendo también un entorno propicio para detectar necesidades en el ámbito de los participantes y dar respuestas a las mismas.

El rol que juega la sociedad en la correcta separación y disposición de estos materiales, obligan a dedicar tiempo y esfuerzos a actividades educativas y de difusión como un tema central de la gestión de RCD. Por otro lado, generar acciones con el sector productivo local que permitan reducir la huella ecológica de los materiales relacionados con la construcción, y por último, contribuir al mejoramiento del hábitat a partir de optimizar recursos materiales y humanos en entornos solidarios por medio de la reutilización de RCD.

La identificación de residuos, la separación en origen y recolección, son fases prioritarias en el proceso de gestión de los RCD. Actualmente en Argentina, no hay una Ley que regule a los generadores de estos. En este sentido, alcanzar una gestión sostenible de los RCD requiere efectivizar un cambio cultural y normativo, siendo para ello imprescindible lograr una comunicación eficiente y la apropiación de ese objetivo por parte de una amplia cantidad de actores.

Teniendo en cuenta las necesidades no satisfechas de amplios sectores de la sociedad en relación al acceso a un hábitat digno, la Universidad en general y la Facultad de Arquitectura en particular no pueden omitir su participación proponiendo estrategias que tiendan a optimizar el uso de los recursos, en pos de dar respuesta a esta problemática, y prioritariamente en este contexto pandémico contribuir con su acción al cuidado de medioambiente, altamente comprometido por una actividad directamente vinculada a su quehacer, como es la construcción de ciudad.

La pandemia del COVID-19 está afectando principalmente a la población de los grandes núcleos urbanos y las zonas más pobladas, donde su propagación es mayor. En este marco, identificando la crisis como una oportunidad de cambio, surge la siguiente pregunta: ¿qué ciudades queremos construir tras la pandemia? Las nuevas formas de habitar llevan a reflexionar sobre la arquitectura y sobre todo a repensar las ciudades que habitamos. 


\section{METODOLOGÍA}

Se parte de la concepción de que el problema de los RCD es un tema complejo de resolver e involucra a todos los sectores poblacionales, con distintos actores, roles y objetivos cada uno. Es por ello que requiere del diseño de diversas estrategias metodológicas y de un equipo diverso para abordar la problemática.

El grupo integrante del proyecto, está compuesto por profesores titulares y adjuntos, ayudantes de curso diplomado del área técnica de la Facultad, estudiantes y graduados. En su mayoría pertenecen al Laboratorio de Tecnología y Gestión Habitacional (LATEC), donde en el último año dado el contexto actual, se han generado cursos virtuales, de capacitación en registro, representación analógica y digital, nuevas tecnologías de diseño asistido, por lo que se cuenta con recursos humanos formados para las actividades.

En cuanto al sector productivo de la construcción, el vínculo se establece a través de la Fundación Espacio Ctibor promoviendo la conexión con la innovación tecnológica, la investigación y el desarrollo de la industria ladrillera y su vinculación con la comunidad local y el ámbito educativo.

En este punto, la estrategia metodológica de este proyecto está enmarcada dentro de la planificación participativa, sobre las que el equipo del LATEC ha desarrollado una sólida experiencia que se centra en generar vínculos entre recursos y necesidades. En este contexto, se adecuaron las actividades del proyecto a un formato virtual:

1) Reuniones virtuales de coordinación y programación de actividades con el equipo del Proyecto y los entes vinculados.

2) Elaboración de un cuadernillo informativo referente al uso de los materiales recuperados y los beneficios ecológico, económico y social en la reutilización de los mismos. Diseño de material gráfico y didáctico para el desarrollo de los talleres de capacitación (folletos, posters, material audiovisual, videos, etc.).

3) Talleres de capacitación virtual sobre el uso eficiente de los materiales intervinientes y patologías de la construcción derivadas de la mala ejecución de las tareas, dirigidos a los destinatarios directos del proyecto. Los talleres se dictan en conjunto con el equipo de proyecto y las cátedras, tales como Procesos Constructivos I, II y III Cremaschi-Saenz, Estructuras I y II Farez-Lozada, Producción de Obras Cremaschi, Instalaciones I y II Lloberas-Toigo, a las cuales pertenecen los docentes participantes del proyecto, actualmente en funcionamiento en todos los entornos virtuales a su alcance. A continuación se detallan los contenidos de los talleres:

- Charlas de presentación de contenido y conceptos básicos para el reconocimiento de intereses, potencialidades y dificultades para el desarrollo de la capacitación.

- Envolventes de la vivienda, mencionando función, tipos y características de las más adecuadas para la escala, modo de ejecución.

- Patologías habituales de las envolventes, prevención y/o reparación de las mismas con análisis de casos y debate participativo.

- Visita guiada virtual al Museo del Ladrillo y su fábrica, a través del vínculo con la Fundación Espacio Ctibor.

4) Evaluación del destino de la donación de materiales disponibles y de las necesidades detectadas, fortaleciendo el vínculo con el sector productivo que acompaña el proyecto.

5) Sistematización de la experiencia. Registro en distintos formatos de la experiencia de los talleres y encuentros realizados. Edición de un material escrito para divulgar la experiencia y sistematizar el proyecto. 
La extensión en Pandemia: la experiencia del Corralón Solidario

Gustavo Cremaschi, María Elisa Cremaschi, María Eugenia Luna y Nelly Lombardi

En un segundo encuentro, se desarrolló el tema de envolventes de la vivienda, mencionando función, tipos y características más adecuadas para la escala, acompañado de su modo de ejecución. Por otro lado, los contenidos del tercer encuentro, se centraron en las patologías habituales de las envolventes, prevención y/o reparación de las mismas, se analizaron casos y se cerró con un debate participativo (Fig. 2)

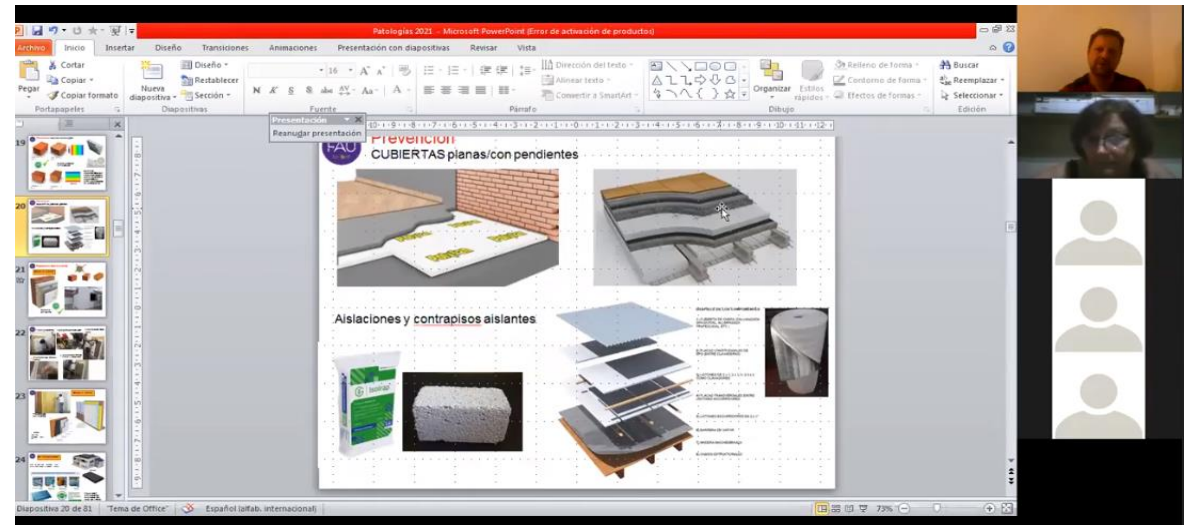

Fig.2. Encuentros de los talleres de capacitación virtual. Fuente: Elaboración propia. 2021.

El cuarto encuentro, se realizó una visita virtual al Museo del Ladrillo (Fig. 3). La actividad fue concretada en conjunto con docentes, graduados/as y estudiantes, a través del vínculo con la Fundación Espacio Ctibor. Durante el encuentro interactivo los participantes tuvieron la oportunidad de abordar diferentes salas temáticas, donde se exhibió el pasado y el presente de la cerámica, su tecnología, usos y propiedades para una mejor eficiencia de las envolventes.
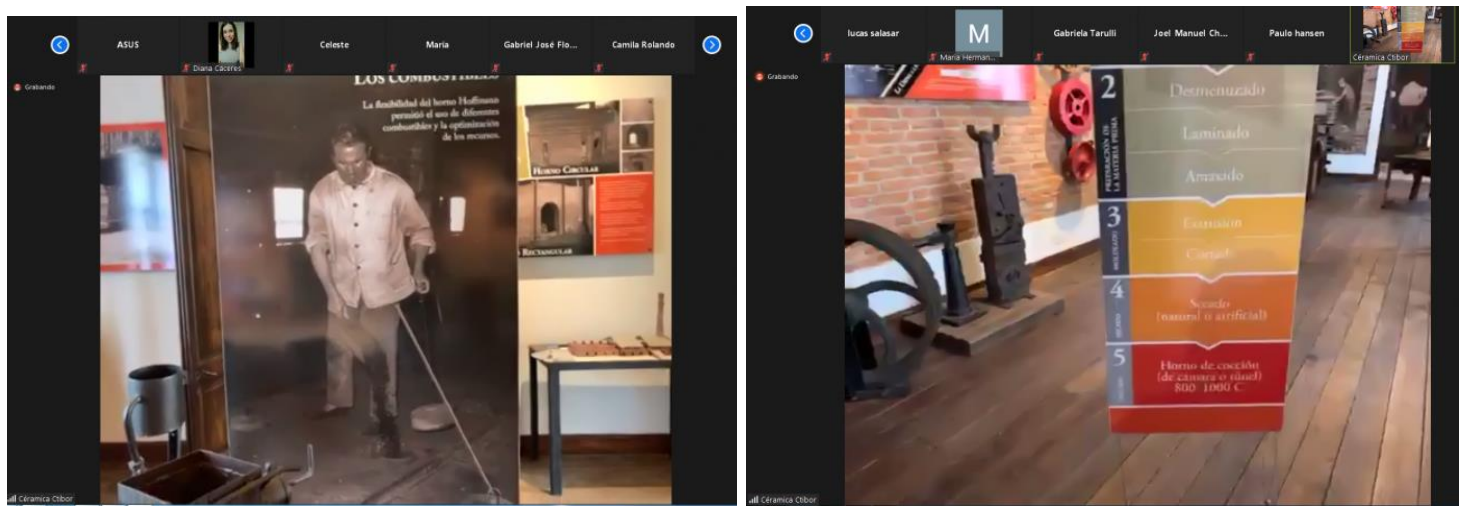

Fig.3.Visita virtual Museo del Ladrillo. Fuente: Elaboración propia. 2021.

Los estudiantes participantes del proyecto colaboraron en el registro y la comunicación del avance del proyecto, acompañando a los docentes en las jornadas de trabajo virtual. Para concientizar y difundir la temática se elaboraron charlas, cartillas, folletos, banners y audiovisuales informativos referentes al uso de los materiales recuperados, su importancia y beneficios tanto ecológico como económico y social.

Por otro lado, las redes sociales forman parte de la transformación digital de los últimos años y se han llegado a instalar en todos los ámbitos de la vida, incluidos la educación, la investigación y la transferencia de conocimiento. Conscientes de eso, el proyecto buscó potenciar dichas herramientas virtuales (Instagram y Facebook), como el canal de difusión de información más inmediato e interactivo para los alumnos, docentes y comunidad en general (Fig.4). 

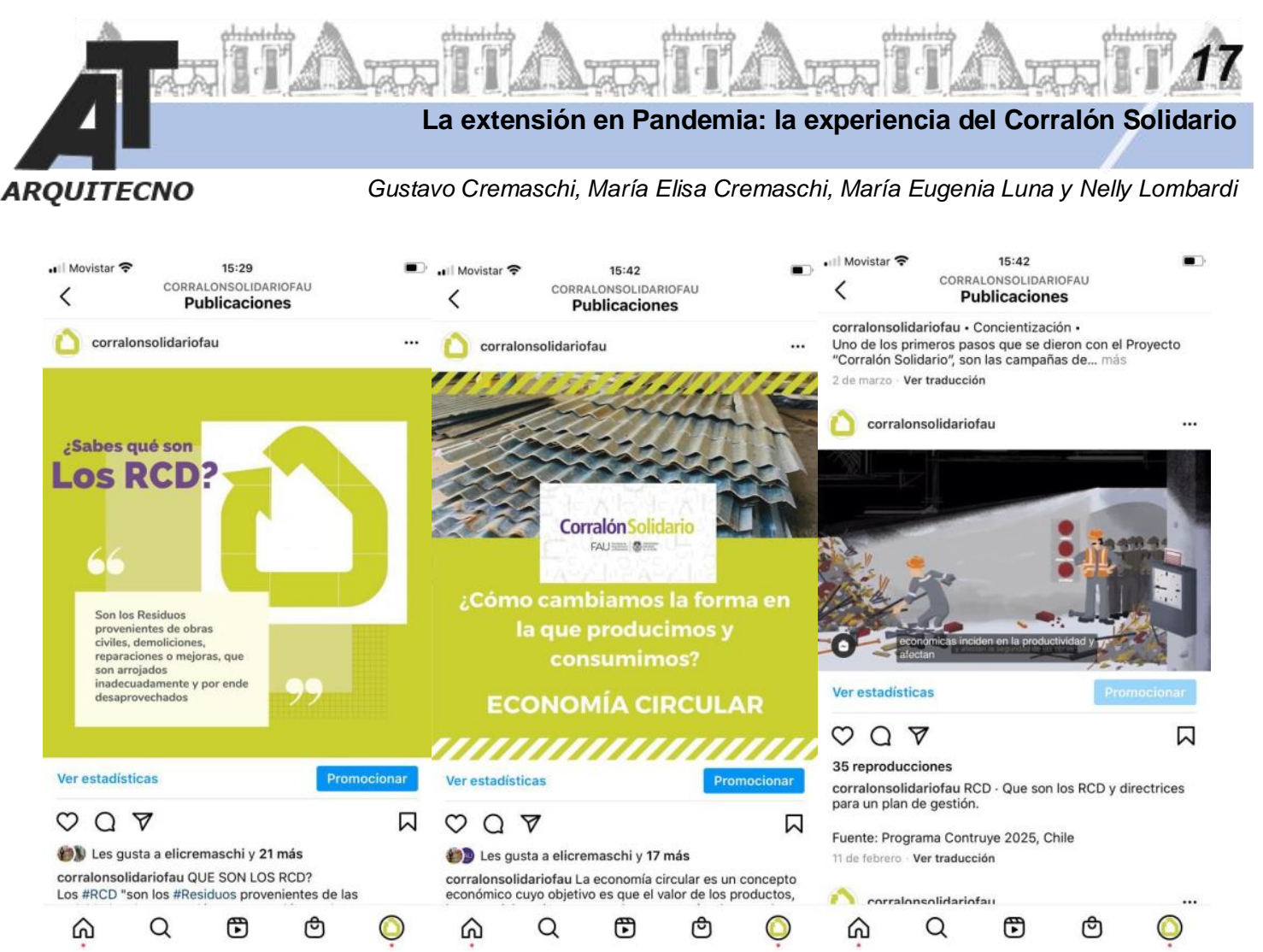

Fig.4. Visualización en las redes sociales. Fuente: Elaboración propia. 2021.

La próxima fase del proyecto involucra a los alumnos de escuela de nivel inicial, primario y secundario, con actividades de sensibilización sobre los efectos de la huella ecológica y los RCD, buscando estrategias que faciliten directamente:

- Desarrollo sostenible: el buen uso y el cuidado de los recursos que tenemos en la actualidad para que no perjudique a generaciones futuras.

- Mayor conocimiento de la naturaleza: una mayor información y conocimiento sobre el medio natural

- Introducción a conceptos básicos como reciclaje: no solo como una forma de ahorro energético, sino también en dar una segunda vida a las cosas.

\section{CONCLUSIONES}

Las mejores sociedades son aquellas que son sostenibles, justas y democráticas. He aquí la importancia de dotar a la comunidad no solo de conocimientos lingüísticos o matemáticos, sino también motivarlos y capacitarlos para satisfacer sus necesidades sin depredar el entorno que habitan. Debemos aprender a vivir en un mundo condicionado por el cambio climático, la crisis ecosistémica y la disposición limitada de energía y materiales.

La FAU UNLP, consciente de esta necesidad no puede omitir su participación proponiendo estrategias que tiendan a optimizar el uso de los recursos, en pos de dar respuesta a esta problemática, y a la vez contribuir con su acción al cuidado de medioambiente, altamente comprometido por una actividad directamente vinculada a su quehacer, como es la construcción de ciudad. Pero estos propósitos deben desarrollarse de forma creativa, vinculando sectores, optimizando procesos, convocando a las instituciones privadas y estatales en sus distintos niveles, de manera de crear conciencia y garantizar su perdurabilidad en el tiempo. En este sentido, el proyecto apunta a un cambio de paradigmas centrado en la sinergia, entre rezago y reutilización, deshecho y aprovechamiento, necesidad y solidaridad. Asimismo propone una facultad permeable, vinculada con el medio, sus necesidades y sus potencialidades, con un 
diálogo fructífero, bidireccional, capaz de interpretar su rol social y dar respuestas para mejorar la calidad de vida de la comunidad.

Una comunidad que se encuentra inmersa en un contexto de incertidumbre y amenazada por el avance de la pandemia. Desde este Proyecto se busca transformar la amenaza en oportunidad, la oportunidad de hacer cosas que antes no se hubiesen pensado o creado:

"La crisis es necesaria para que la humanidad avance. Solo en momentos de crisis, surgen las grandes mentes".

Albert Einstein.

\section{BIBLIOGRAFÍA}

Asociación Española de Reciclaje de RCD (2017). Informe de Producción y Gestión de RCD en España Periodo 2011-2015. España. Disponible en web: https://rcdasociacion.es/images/documents/Informe-RCDA-11-15.pdf

Commoner, B. (1992). En paz con el planeta. Barcelona. Ed. Crítica.

Comunidad de Madrid (2015). Plan de gestión de residuos de demolición y construcción. España. Disponible en web: http://www.madrid.org/es/transparencia/sites/defaultfiles/regulation/documents/5 plan d e gestion de residuos de construccion y demolicion.pdf

Del Val, A. (1997). El libro del Reciclaje. 3ª Edición. Barcelona. Ed. RBA Libros.

Edwards, B. (2009) Guía Básica de la Sostenibilidad. Segunda edición revisada y ampliada. Londres. Ed.G. Gilli.

Hernandez Pezzi, C. (2007). Un Vitruvio Ecológico. Principio y práctica del proyecto arquitectónico sostenible. Barcelona. Ed.G. Gilli.

Lynch, K.; Southworth, M. (2005) Echar a Perder: un análisis del deterioro. ISBN: 84-252-2044-0

España. Editorial Gustavo Gili SL.

Mercante, I. T. (2007). Caracterización de residuos de la construcción. Aplicación de los índices de generación a la gestión ambiental. Revista Científica de Primavera UCES, 24.

Varón, L., Sierra, D., \& Bedoya, L. (2011). Indural: un aporte significativo a la producción más limpia y la construcción sostenible. Rev. P+L [online]. 2011, vol.6, n.1, pp.128-135. ISSN 1909-0455. España. Editorial Lasallista.

Wolff, G. (2017). Protocolo de Gestión de residuos de construcción y demolición en la Unión Europea. Madrid. Publicado por European Commission. 


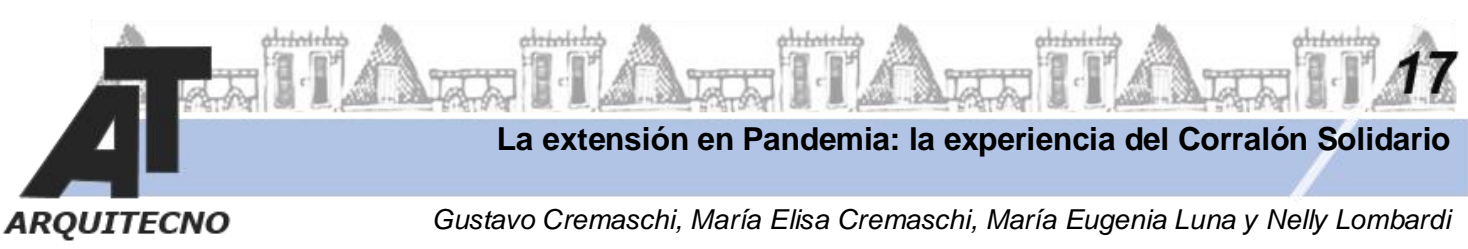

Fuentes Legales:

Real Decreto 105/2008. Regulación de la producción y gestión de residuos de construcción y demolición. España. Disponible en web: http://www.euskadi.eus/informacion/registrode-produccion-y-gestion-de-residuos/web01-a2inghon/es/ 